\title{
JOURNAL OF CLINICAL AND EXPERIMENTAL INVESTIGATIONS
}

\section{Live Birth Rates After Assisted Reproductive Techniques of Women Aged 42 and Over}

\author{
Gulnaz Sahin ${ }^{1^{*}}$, Ferruh Acet ${ }^{1,2}$, Ege Nazan Tavmergen Goker ${ }^{1,2}$, Erol Tavmergen ${ }^{1,2}$
}

\begin{abstract}
${ }^{1}$ Ege University Family Planning and Infertility Research and Practice Center, Bornova, Izmir, Turkey

${ }^{2}$ Department of Obstetrics and Gynecology, Ege University Faculty of Medicine, Ankara cad., Bornova, Izmir, Turkey
\end{abstract}

\section{Correspondence:}

Gulnaz Sahin

Address: Ege University Family Planning and Infertility Research and Practice Center, Bornova, Izmir, Turkey

Email: gsahini@msn.com

Received: 27.01.2021,

Accepted: 05.08.2021

https://doi.org/10.29333/jcei/11214

\begin{abstract}
Objective: Increased oocyte aneuploidy rates, along with the quantitative decrease of oocytes, are the main consequences of reproductive aging. We aimed to analyze assisted reproductive technique (ART) outcomes of women at extremely advanced age from a single tertiary IVF center.

Material and Methods: This retrospective cohort study included women aged $>42$ years and underwent oocyte pickup between 2015 and 2020, in a tertiary IVF center. Reproductive outcomes of 178 women who underwent oocyte pickup and subsequent fresh and/or frozenthawed autologous embryo transfer (ET) were analyzed.

Results: A total of 261 oocyte pickup cycles from 178 women aged $>42$ years performed during the study period. The mean age of women was $44.5 \pm 1.4$. Overall, 118 women $(66.3 \%)$ underwent either autologous fresh and/or frozen embryo transfer cycles, and 60 women (33.7\%) could not reach to embryo transfer. Clinical pregnancy and live birth rates were $(5.0 \%$, $1.1 \%$ per patient), (5.8\%, $1.3 \%$ per embryo transfer) and (3.4\%, $0.7 \%$ per cycle), respectively. Those patients who had live birth were 43 years old at oocyte retrieval, and their pregnancies were obtained from frozen-thawed embryo transfer.

Conclusion: The pregnancy and live birth rates are extremely low for those patients at a very advanced age; however, there might be extreme cases with a successful result. Availability of supernumerary embryos for future frozen-thawed transfers might give a chance these patients to conceive. As with all infertile patients, an individualized approach should be taken into account in advanced age groups.
\end{abstract}

Keywords: aging, assisted reproductive techniques, pregnancy rate, live birth

\section{INTRODUCTION}

The declining number and quality of oocytes by advanced female age is a wellknown phenomenon. The success of assisted reproductive techniques (ART) is strictly dependent on the female age. Age is the most established predictor of IVF outcomes [1,2]. Increased oocyte aneuploidy rates, along with the quantitative decrease of oocytes, are the main consequences of reproductive aging. This evidence is also mirrored in the ART outcomes.

Delaying childbirth has been a common problem worldwide. Women's attempts to conceive at advanced reproductive ages are increasing [3]. According to the US national data from 2017, $20.7 \%$ of women who underwent an ART cycle were $>40$ years old, and $40 \%$ of these women was used their oocytes [4]. The reported live birth rates (LBR) were $8.1 \%$ for this age group [4]. Fertility treatment for the advanced age group remains difficult and controversial. Some European countries have female age limits for legal access to ART such as 45 years old in Denmark and 51 years old in Bulgaria [5].

ART is believed to offer the best chance for pregnancy in advanced reproductiveaged women [6]. However, ART treatments' success for these poor prognostic women remains constantly low because of their already reduced ovarian reserve and/or high risk of embryonic aneuploidies leading to implantation failure or miscarriage.

Several authors have evaluated the ART 
outcomes of women aged 40 and over [6-10]. Few also analyzed specifically more advanced age groups such cutoff as 43-45 years of age [11-13]. In a study from Korea, including more than 2000 cycles in 1049 women aged 40 and over, the decrease in cumulative LBR's was clearly shown from $12.9 \%$ to $0.7 \%$ of the women aged 40 to $\geq 45$ [7]. The cumulative LBR's were reported as $2.4 \%, 2.1 \%$, and $0.7 \%$ at age of 43,44 , and 45 years old. Similarly, a significant decline in cumulative LBR's has been reported in women $\geq 43$ years compared those aged 41 and 42 years in previous studies [8,9]. Gleicher et al. emphasized the age 43 is a breakpoint for IVF outcomes [14]. The chance of the live birth (LB) with an ART treatment is at extremely lower rates, specifically age over 42 years old. On the other hand, there are reports of patients with extremely advanced age and successfully achieved LB after ART using autologous oocytes [15-18]. The decision to proceed or not to proceed to an ART treatment should be based on the patient's findings and their own decision after detailed information on the potential risks and success rates of ART treatment.

Evaluating the chance of LB with their gametes in an ART program is pivotal to counsel advanced aged patients appropriately. We aimed to evaluate the ART outcomes and LB chances of women aged $>42$ years who underwent ART treatment in our university-based tertiary IVF center.

\section{MATERIALS AND METHODS}

Women aged $>42$ years and underwent oocyte pickup between 2015 and 2020 in our tertiary IVF center were included in this retrospective cohort study. Based on these criteria, a total of 261 oocyte pickup (opu) cycles of 178 women aged $>42$ during the procedure were found from the IVF database and included in the study. Subsequent fresh and/or frozen embryo transfer cycle outcomes were evaluated. The variables included clinical and IVF outcomes were retrieved from patient records and the IVF database. The inclusion criteria were; female age $>42$ years during the oocyte pickup procedure.

Controlled ovarian stimulation was performed using exogen gonadotropins with the antagonist protocol. Human chorionic gonadotropin (hCG) was used for ovulation trigger when the leading follicle reached $18 \mathrm{~mm}$ in diameter. Oocyte pickup was performed 34-36 hours after the ovulation trigger. ICSI was used as the fertilization method. A maximum of two embryos was transferred on day 2 to 5 . The luteal phase was supported using vaginal micronized progesterone with single dose rechCG on the fifth day of embryo transfer. In case of available surplus embryos after a fresh transfer, vitrified for another thawed cycle. In some cycles, fresh transfer was not performed, and embryos were vitrified, aiming for preimplantation aneuploidy screening or other reasons. Endometrial preparation was performed either with a modified natural cycle or an artificial preparation cycle for thawed embryo transfers.
Table 1. Characteristics of the AMA group

\begin{tabular}{|c|c|}
\hline Number of patients & 178 \\
\hline No of opu cycles & 261 \\
\hline Age $^{a}$ & 44.5(1.4) (min:43-max:49) \\
\hline \multicolumn{2}{|l|}{ Infertility etiologies (\%) } \\
\hline Unexplained & 82.1 \\
\hline Male factor & 16.8 \\
\hline Tuboperitoneal factor & 1.1 \\
\hline No of Previous IVF cycles ${ }^{\mathrm{a}}$ & $1.0(1.5)(\min : 0-\max : 7)$ \\
\hline
\end{tabular}

Clinical pregnancy was defined as an ultrasonographic visible gestational sac in the uterine cavity, and live birth was defined as the birth of an infant after $24^{\text {th }}$ weeks of gestation. Miscarriage was defined as a clinical pregnancy loss. Ethical approval was obtained from Institutional Ethics Committee.

\section{Statistical Analysis}

The main outcome measure was the cumulative live birth rates of the patients. Secondary outcomes were clinical pregnancy rates, the number of total and mature oocytes, fertilization rates, percentages of successful embryo transfer cycles, and miscarriage rates. Continuous variables were given as mean (SD) and ranges, categorical variables were expressed as frequencies and percentages (\%). The binary logistic regression model was used to evaluate factors that may associate with the probability of clinical pregnancy and live birth (age, AMH level, the total number of oocytes after the first ovarian stimulation). Statistical significance was considered at $p$-value $<0.05$. Multiple regression analysis was executed if multiple factors were found significant. The IBM SPSS software (ver. 25.0, SPSS Inc., USA) was used for the analyses.

\section{RESULTS}

Overall, 261 opu cycles from 178 women aged $>42$ years performed in our tertiary IVF center between 2015-2020, were included in this retrospective study. Patients' age range was 43 to 49 years old, and the mean age (SD) was 44.5(1.4). In this group of patients, male factor (16.8\%) and tuboperitoneal factor (\%1.1) were concomitant infertility etiologies. In the study period, a single opu cycle was performed in 138 patients (\% 77.5) and 40 patients (\%22.5) had recurrent cycles (mean:2.4(1.6), range:1-9) Characteristics of the patients are shown in Table 1.

The mean (SD) number of oocytes retrieved was; 2.9 (2.6) (range: $0-13$ ). In total, no oocyte was obtained in 24 cycles and no mature oocytes in 12 cycles. Therefore, there were a total of 225 cycles $(86.2 \%)$ obtaining at least one oocyte for the ICSI procedure. The mean number of oocytes that underwent ICSI was 2.2 (1.9) (range:1-10) with a fertilization rate of $70.0 \pm 36.5 \%$. Of those 261 cycles; 137 cycles ended up with fresh embryo transfer (ET) whereas in 87 cycles there were no embryos available for transfer, and the embryos of the remaining 37 cycles were cryopreserved. Within overall $16 / 261$ cycles $(6.1 \%)$, had surplus embryos which were 
Table 2. Cycle outcomes of AMA group ( $n=178$ women)

\begin{tabular}{|c|c|}
\hline No of opu cycles & 261 \\
\hline Total gonadotropin used (IU) ${ }^{\mathrm{a}}$ & 2533(903) \\
\hline Peak E2 level $(\mathrm{pg} / \mathrm{ml})^{\mathrm{a}}$ & $646(472)$ \\
\hline No of oocyte retrieved ${ }^{a}$ & $2.9(2.6)$ \\
\hline No of mature oocytes ${ }^{a}$ & $2.2(1.9)$ \\
\hline No of fertilized oocytes ${ }^{a}$ & $1.5(1.5)$ \\
\hline Fertilization rate (\%) & $70.0(36.7)$ \\
\hline \multicolumn{2}{|l|}{ No of 2PN's } \\
\hline Fresh ET cycles (n,\%) & $137(52.5 \%)$ \\
\hline $\begin{array}{l}\text { Cycles without embryos available for } \\
\text { transfer }(n, \%)\end{array}$ & $87(33.3 \%)$ \\
\hline All freeze cycles $(n, \%)$ & $37(14.2 \%)$ \\
\hline Clinical pregnancy/per cycle (\%) & 3.4 \\
\hline Clinical pregnancy/per ET (\%) & 5.8 \\
\hline Clinical pregnancy/per patient (\%) & 5.0 \\
\hline Live birth/per cycle (\%) & 0.7 \\
\hline Live birth/per ET (\%) & 1.3 \\
\hline Live birth/per patient (\%) & 1.1 \\
\hline
\end{tabular}

vitrified after fresh embryo transfer. Preimplantation genetic diagnosis for aneuploidy screening (PGD-A) was intended in $10 / 178(5.6 \%)$ patients in our study population. Of those, no embryo was developed for analysis in a patient. No embryos were found normal in two patients, the remaining
7 had no clinical pregnancy after the transfer of embryos analyzed.

Subsequent autologous fresh (n:137 cycles) and/or frozen-thawed (n:16 cycles) embryo transfer cycles were performed in 118 women (66.3\% of the total group). Twelve pregnancies were obtained (biochemical:3, miscarriage:7, and live birth:2). Cumulative reproductive outcomes, including fresh and frozen ET cycles, were analyzed. The cumulative clinical pregnancy rate was $3.4 \%$ and $5.0 \%$, per opu cycle and per patient. The LBR's was, $0.7 \%$ and $1.1 \%$, per opu cycle and per patient, respectively (Table 2 ). Those patients who gave live birth both were 43 years old at oocyte retrieval, and their pregnancies were developed following frozen-thawed ET.

Factors that may predict the cumulative clinical pregnancy and live birth (age, AMH level, total number of oocytes from the first cycle) were evaluated by binary and multiple logistic regression models. The mean AMH levels were $0.68(0.8) \mathrm{ng} / \mathrm{ml}$ (range: $0.01-6.8)$ and were available in 135 women ( $75.8 \%$ of the total group). Regression analysis showed among the variables, the number of oocytes was independently associated with both clinical pregnancy and LB's (Tables 3 and 4). As expected, an increase in oocyte number was significantly associated with likelihood of $\mathrm{CP}$ and LB in this group.

\section{DISCUSSION}

This study explored the probability of live birth after ART in women aged $>42$ years with their oocytes. Our results

Table 3. Binary Logistic Regression Models for Single Variables to Predict Cumulative Clinical Pregnancy

\begin{tabular}{cccc}
\hline \multirow{2}{*}{ Variables } & \multicolumn{2}{l}{ Single Variables } \\
\cline { 2 - 4 } & $\mathbf{p}$ & OR & $\mathbf{9 5 \%}$ C.I. \\
\hline Age & $0.040^{\mathrm{a}}$ & 0.401 & $0.167-0.959$ \\
\hline AMH & $0.023^{\mathrm{a}}$ & 2.084 & $1.106-3.928$ \\
\hline Number of oocytes & $<0.001^{\mathrm{a}}$ & 1.606 & $1.275-2.023$ \\
\hline Variables & $\mathrm{p}$ & Final Model & \\
\hline Age & 0.162 & OR & $95 \%$ C.I. \\
\hline AMH & 0.693 & 0.438 & $0.137-1.395$ \\
\hline Number of oocytes & $0.027^{\mathrm{a}}$ & 1.159 & $0.557-2.410$ \\
\hline
\end{tabular}

a $\mathrm{p}<0.05$, statistically significant; C.I.: Confidence Interval; OR: Odds ratio

${ }^{b}$ Multiple logistic regression final model was executed on all these variables, included together in the model

Table 4. Binary Logistic Regression Models for Single Variables to Predict Cumulative Live Birth

\begin{tabular}{cccc}
\hline \multirow{2}{*}{ Variables } & \multicolumn{3}{c}{ Binary Logistic Regression Models for Single Variables } \\
\cline { 2 - 4 } & $\mathbf{p}$ & $\mathbf{O R}$ & $\mathbf{9 5 \%}$ C.I. \\
\hline Age & 0.993 & - & - \\
\hline AMH & 0.063 & 2.145 & $0.960-4.792$ \\
\hline Number of oocyte & $0.030^{\mathrm{a}}$ & 1.696 & $1.052-2.732$ \\
\hline
\end{tabular}

a $p<0.05$, statistically significant; C.I.: Confidence Interval; OR: Odds ratio 
suggested that live birth rates are extremely low for those patients. The real success of the ART treatments is the birth of a healthy infant. Specific counseling on the ART success rates for advanced reproductive-aged women who have a low chance of live birth is pivotal in clinical practice. Most of those women do not sufficiently know the age-related decline of the pregnancy and live birth rates of ART treatments and also medical risks of delayed childbearing. The center's data results on this specific issue may help patients to realize their expectations.

In a study by Devesa et al. (2018), cumulative LBR's including fresh and frozen ET's after single stimulation cycles of 4570 infertile women aged $\geq 38$ years were evaluated according to the age and number of oocyte yields [19]. In their study, the most prominent decrease in LBR's was shown at $42-43$ years, and the cumulative LBR was the only $\% 1.2$ in women aged $\geq 44$ years. Authors also reported that a higher number of oocytes related with higher cumulative LBR's more evidently up to 41 years old, but without clear benefit beyond 44 years of age [19]. Substantial contribution of cryopreserved embryos to cumulative LBR's was shown until women aged 42 years. The observed benefit was limited to $3 \%$ and $0 \%$ for $42-43$ years and aged beyond 44 years, respectively. The majority of the cryopreservation method in that study was slow freezing; thus, the authors emphasized that the results need to be interpreted with caution. The authors also concluded that IVF with own oocytes should be discouraged in women aged over 43 years based on their results.

Spandorfer et al. evaluated the IVF outcomes of women age between 45-49. The authors reported that, of the 231 initiated IVF cycle, $2.2 \%$ ended with delivery, and only patients at 45 years of age and with good response have been achieved this result [11]. A decade later, in another retrospective study from the same center, Gunnala et al. analyzed 1078 fresh autologous IVF cycles of women aged 45 and over, without preimplantation genetic diagnosis (PGD), including a 20 -year period. [12]. The LBR per cycle was $2.9 \%$ at 45 years and $0.5 \%$ at 46 years group and the oldest age who delivered was 46 in this group. The authors noticed high pregnancy loss rates $(82.3 \%)$. They concluded that live birth with autologous IVF in women aged over 46 is unlikely.

The increased embryo aneuploidy rates by advanced women age are well known to cause the age-related decline in pregnancy rates [20]. Mechanisms of age-related oocyte aneuploidies and impairment of oocyte competence have been discussed in detail [21-23]. Preimplantation genetic screening has been suggested for advanced age women as a logical approach for improving ART success by selecting euploid embryos [24,25]. Ubaldi et al. (2017) evaluated results of PGD-A of 137 women aged $\geq 44$ years with clinical outcomes [25]. No euploid blastocysts for patients aged over 45 were reported. The delivery rate of $10.0 \%$ per cycle for the age of 44 and $2.6 \%$ for the age of 45 was shown after PGD-A in their study group. The authors suggested the use of PGDA for these women with good ovarian reserve [25].

However, low oocyte yield is a limiting factor for PGD in advanced age women. To overcome this situation, embryo or oocyte pooling with several consecutive conventional stimulation cycles or both follicular and luteal phase stimulation in the same cycle has been proposed [26-28]. Nevertheless, limited data on this approach's efficiency and cost-effectiveness has been noticed $[19,25,29]$. In our study population, PGD-A was performed only in $5.6 \%$ of the patients, possibly due to the procedure's cost and time need.

In a recent study, Warshaviak et al. compared morphokinetic parameters of embryos from women aged $>42$ years ( 51 women, 496 embryos) and $<38$ years $(71$ women, 653 embryos), during the first 3 days of development, by time-lapse microscopy [30]. No significant differences in the evaluated parameters except tendency toward developmental arrest until the 8-cell stage in the advanced age group were reported. The authors suggested, a possible significant delay could be seen in later morphokinetic parameters if the culture period extended to the blastocyst stage. In their group, the LBR's were $2 \%$ for women aged $>42$ years old, with significantly lower than the younger group (27\%). Gleicher et al. specifically reported the pregnancy outcomes of the extremely advanced age women (43 to 51 years) following IVF with autologous oocytes during the 2014-2016 periods [13]. The LBR's were $1.1 \%$, $0.43 \%$, and $1.3 \%$ per cycle for the yearly bases, respectively. They have reported two pregnancies with autologous IVF in the two oldest women aged 47 in the presented group. One of them ended with live birth and other loss of pregnancy with complex aneuploidy. They concluded that in selected women at very advanced age with a good ovarian reserve and with individualized managing, IVF with own oocytes can be an option.

Our study presents, pregnancy outcomes of women aged $>42$ years following ART from a single tertiary IVF unit. This study's strength is evaluating cumulative pregnancy outcomes of a significant number of advanced age women from a single IVF unit including the last five-year period. Although its retrospective nature is a limitation, the study results are expressing the daily clinical practice.

In conclusion, our study results showed extremely low live birth rates in women aged $>42$ years. However, there might be cases with a successful result. Good ovarian response and availability of supernumerary embryos for future frozen-thawed transfers might give a chance for these patients to conceive. As with all infertile patients, an individualized approach should be taken into account in advanced age groups.

Author contributions: GS: contributed to the study design and conducted to the data collection, analysis and writing the manuscript, FA: contributed to the data collection, data analysis and literature search, EG: contributed to the interpretation of the data and critical 
revision of the manuscript, ET: contributed to the interpretation of the data and critical revision of the manuscript.

Funding: No funding source is reported for this study.

Acknowledgements: The authors acknowledge Assoc. Prof. Asli Suner for her help on statistical analysis.

Declaration of interest: No conflict of interest is declared by authors.

\section{REFERENCES}

1. Vaegter KK, Lakic TG, Olovsson M, Berglund L, Brodin T, Holte J. Which factors are most predictive for live birth after in vitro fertilization and intracytoplasmic sperm injection (IVF/ICSI) treatments? Analysis of 100 prospectively recorded variables in 8,400 IVF/ICSI single-embryo transfers. Fertil Steril. 2017;107:641-8. doi: 10.1016/j.fertnstert.2016.

2. McLernon DJ, Steyerberg EW, Te Velde ER, Lee AJ, Bhattacharya S. Predicting the chances of a live birth after one or more complete cycles of in vitro fertilisation: population based study of linked cycle data from 113873 women. BMJ. 2016;355:i5735. doi: 10.1136/bmj.i5735.

3. Schmidt L, Sobotka T, Bentzen JG, Nyboe Andersen A; ESHRE Reproduction and Society Task Force. Demographic and medical consequences of the postponement of parenthood. Hum Reprod Update. 2012;18:29-43. doi: 10.1093/humupd/dmr040.

4. CDC: Centers for Disease Control and Prevention, National Center for Chronic Disease Prevention and Health Promotion. Division of Reproductive Health. Available at: http://nccd.cdc.gov/drh_art (Accessed: 23 December 2020).

5. Calhaz-Jorge C, De Geyter CH, Kupka MS, et al. Survey on ART and IUI: legislation, regulation, funding and registries in European countries: The European IVFmonitoring Consortium (EIM) for the European Society of Human Reproduction and Embryology (ESHRE). Hum Reprod Open. 2020;2020:hoz044. doi: 10.1093/hropen/hoz044.

6. Auyeung A, Klein ME, Ratts VS, Odem RR, Williams DB. Fertility treatment in the forty and older woman. J Assist Reprod Genet. 2001 Dec;18(12):638-43. doi: 10.1023/a:1013159116180.

7. Kim HO, Sung N, Song IO. Predictors of live birth and pregnancy success after in vitrofertilization in infertile women aged 40 and over. Clin Exp Reprod Med. 2017;44:111-7. doi: 10.5653/cerm.2017.44.2.111

8. Klipstein S, Regan M, Ryley DA, Goldman MB, Alper $\mathrm{MM}$, Reindollar RH. One last chance for pregnancy: a review of 2,705 in vitro fertilization cycles initiated in women age 40 years and above. Fertil Steril. 2005;84:43545. doi: 10.1016/j.fertnstert.2005.02.020.
9. Serour G, Mansour R, Serour A, et al. Analysis of 2,386 consecutive cycles of in vitro fertilization or intracytoplasmic sperm injection using autologous oocytes in women aged 40 years and above. Fertil Steril. 2010;94:1707-12. doi: 10.1016/j.fertnstert.2009.09.044.

10. Reljič M, Lovrec VG. Predictive Factors for Live Birth in Autologous in Vitro Fertilization Cycles in Women Aged 40 Years and Older. Zdr Varst. 2019;58:173-8. doi: 10.2478/sjph-2019-0022.

11. Spandorfer SD, Bendikson K, Dragisic K, Schattman G, Davis OK, Rosenwaks Z. Outcome of in vitro fertilization in women 45 years and older who use autologous oocytes. Fertil Steril. 2007;87:74-6. doi: 10.1016/j.fertnstert.2006.05.081.

12. Gunnala V, Irani $M$, Melnick A, Rosenwaks Z, Spandorfer S. One thousand seventy-eight autologous IVF cycles in women 45 years and older: the largest single-center cohort to date. J Assist Reprod Genet. 2018;35:435-40. doi: 10.1007/s10815-017-1088-y.

13. Gleicher N, Kushnir VA, Darmon S, Albertini DF, Barad DH. Older women using their own eggs? Issue framed with two oldest reported IVF pregnancies and a live birth. Reprod Biomed Online. 2018;37:172-7. doi: 10.1016/j.rbmo.2018.05.010.

14. Gleicher N, Kushnir VA, Albertini DF, Barad DH. Improvements in IVF in women of advanced age. J Endocrinol. 2016;230:F1-6. doi: 10.1530/JOE-16-0105.

15. Nicopoullos JD, Wren M, Abdalla $H$. Treatment and preservation at the extremes of reproductive age: a case report outlining the ethical dilemmas. J Assist Reprod Genet. 2015;32:1547-50. doi: 10.1007/s10815-015-0551$\mathrm{x}$.

16. Dal Prato L, Borini A, Cattoli M, Preti MS, Serrao L, Flamigni C. Live birth after IVF in a 46-year-old woman. Reprod Biomed Online. 2005;11:452-4. doi: 10.1016/s1472-6483(10)61139-x.

17. Giannaris D, Orfanoudaki E, Zourla A, Salamalekis E. Clinical pregnancy and delivery of a healthy baby by a 44-year-old woman after a successful in vitro fertilization frozen cycle using her own oocytes. Fertil Steril. 2009;91:293.e5-7. doi: 10.1016/j.fertnstert.2007.01.027.

18. Trolice MP. Live birth from a 46-year-old using fresh autologous oocytes through in vitro fertilization. Fertil Steril. 2014;102:96-8. doi: 10.1016/j.fertnstert.2014.04.014.

19. Devesa M, Tur R, Rodríguez I, Coroleu B, Martínez F, Polyzos NP. Cumulative live birth rates and number of oocytes retrieved in women of advanced age. A single centre analysis including 4500 women $\geq 38$ years old. Hum Reprod. 2018;33:2010-7. doi: 10.1093/humrep/dey295 
20. Franasiak JM, Forman EJ, Hong KH, et al. The nature of aneuploidy with increasing age of the female partner: a review of 15,169 consecutive trophectoderm biopsies evaluated with comprehensive chromosomal screening. Fertil Steril. 2014;101:656-63.e1. doi: 10.1016/j.fertnstert.2013.11.004.

21. Capalbo A, Hoffmann ER, Cimadomo D, Ubaldi FM, Rienzi L. Human female meiosis revised: new insights into the mechanisms of chromosome segregation and aneuploidies from advanced genomics and time-lapse imaging. Hum Reprod Update. 2017;23:706-22. doi: 10.1093/humupd/dmx026.

22. Mikwar M, MacFarlane AJ, Marchetti F. Mechanisms of oocyte aneuploidy associated with advanced maternal age. Mutat Res. 2020;785:108320. doi: 10.1016/j.mrrev.2020.108320.

23. Cimadomo D, Fabozzi G, Vaiarelli A, Ubaldi N, Ubaldi FM, Rienzi L. Impact of Maternal Age on Oocyte and Embryo Competence. Front Endocrinol (Lausanne). 2018;9:327. doi: 10.3389/fendo.2018.00327.

24. Schoolcraft WB, Katz-Jaffe MG. Comprehensive chromosome screening of trophectoderm with vitrification facilitates elective single-embryo transfer for infertile women with advanced maternal age. Fertil Steril. 2013;100:615-9. doi: 10.1016/j.fertnstert.2013.07.1972.

25. Ubaldi FM, Cimadomo D, Capalbo A, et al. Preimplantation genetic diagnosis for aneuploidy testing in women older than 44 years: a multicenter experience. Fertil Steril. 2017;107:1173-80. doi: 10.1016/j.fertnstert.2017.03.007.
26. Chatziparasidou A, Nijs M, Moisidou $M$, et al. Accumulation of oocytes and/or embryos by vitrification: a new strategy for managing poor responder patients undergoing pre implantation diagnosis. F1000Res. 2013;2:240. doi: 10.12688/f1000research.2-240.v2.

27. Orris JJ, Taylor TH, Gilchrist JW, Hallowell SV, Glassner MJ, Wininger JD. The utility of embryo banking in order to increase the number of embryos available for preimplantation genetic screening in advanced maternal age patients. J Assist Reprod Genet. 2010;27:729-33. doi: 10.1007/s10815-010-9474-8.

28. Ubaldi FM, Capalbo A, Vaiarelli A, et al. Follicular versus luteal phase ovarian stimulation during the same menstrual cycle (DuoStim) in a reduced ovarian reserve population results in a similar euploid blastocyst formation rate: new insight in ovarian reserve exploitation. Fertil Steril. 2016;105:1488-1495.e1. doi: 10.1016/j.fertnstert.2016.03.002.

29. Vaiarelli A, Cimadomo D, Capalbo A, et al. Preimplantation genetic testing in ART: who will benefit and what is the evidence? J Assist Reprod Genet. 2016;33:1273-8. doi: 10.1007/s10815-016-0785-2.

30. Warshaviak M, Kalma Y, Carmon A, et al. The Effect of Advanced Maternal Age on Embryo Morphokinetics. Front Endocrinol (Lausanne). 2019;10:686 doi: $10.3389 /$ fendo.2019.00686. 